\title{
Diagnostic limitations in the treatment of cerebral aneurysms
}

\author{
Gmeiner $\mathrm{M}^{1}$, Sonnberger $\mathrm{M}^{2}$, Pogady $\mathrm{P}^{1}$ \\ Department of Neurosurgery, Landes-Nervenklinik Wagner-Jauregg, Linz, Austria. \\ matthias.gmeiner@yahoo.com
}

\begin{abstract}
BACKGROUND: Endovascular coiling has become a standard technique in the treatment of cerebral aneurysms. The mechanisms of recurrence are incompletely understood.

METHODS: In our clinical investigations we present three uncommon cases where the pathology at the base might has been underestimated in digital subtraction or magnetic resonance angiography.

RESULTS: In the first clinical study rehemorrhage occurred 11 years after endovascular coiling. Before rehemorrhage occurred, serial magnetic resonance angiographies had revealed a stable situation with only a small base remnant after initial endovascular treatment. In the second clinical study, intraprocedural rupture during endovascular coiling occurred and a residual angiographic occult lesion was detected only during microsurgical clipping. In the third clinical study, we again found a residual lesion during microsurgical clipping.

CONCLUSION: We present three clinical studies were the pathology at the base might has been underestimated in digital subtraction angiography or magnetic resonance angiography. The incidence of angiographic occult residuals is unknown, but their clinical relevance may be important. Beside other mechanisms, these lesions might be the source of aneurysmal regrowth and hemorrhage (Fig. 4, Ref. 27). Text in PDF www.elis.sk. KEY WORDS: endovascular coiling, cerebral aneurysm, base, angiographic occult lesion, neurosurgery.
\end{abstract}

\section{Introduction}

Endovascular treatment has become a standard technique for treating cerebral aneurysms after the International Subarachnoid Aneurysm Trial (ISAT) (1) study. Following its introduction with Guglielmi detachable coils (GDC) in the 1990s, recent developments including modified coils, balloon protection devices, intracranial stents or semipermeable stents have increased the number of endovascular treatable aneurysms (2).

Here, we present two uncommon studies dealing with intraprocedural rupture and late rehemorrhage. Further, we provide a case where a small lesion at the base was found intraoperatively was after being missed in preoperative digital subtraction angiography (DSA).

Although these cases are completely different as to their pathogenesis, detailed evaluation has suggested the base as the source for rebleeding or potential future hemorrhage.

\section{Clinical study 1}

This 67-year-old woman presented with subarachnoid hemorrhage (Fig. 1A) (SAH, clinical Hunt-Hess II, March 2003). DSA

\footnotetext{
${ }^{1}$ Department of Neurosurgery, Landes-Nervenklinik Wagner-Jauregg, Linz, Austria, and ${ }^{2}$ Department of Neuroradiology, Landes-Nervenklinik Wagner-Jauregg, Linz, Austria
}

Address for correspondence: M. Gmeiner, MD, PhD, Department of Neurosurgery, Landes-Nervenklinik Wagner-Jauregg, Wagner-JaureggWeg 15A, 4020 Linz, Austria.

Phone: +41.50.5546228453, Fax: +41.50 .5546225904$ revealed an anterior communicating artery aneurysm (Fig. 1B). This aneurysm was treated with GDCs via the endovascular route and almost complete coiling was achieved. Only decent central filling could be observed (Fig. 1C). The patient recovered well and the control DSA was applied after 6 months. The fundus was completely coiled leaving only a small remnant at the base. Due to the stable situation, we decided to follow the patient with serial magnetic resonance angiographies up to ten years (May 2004, April 2005, May 2009, May 2011, and May 2013). In February 2014 this patient presented with SAH (clinical Hunt-Hess II) again (Fig. 1D). DSA analyses revealed the initial aneurysm coiled with a small remnant at the base and a new 2x3 mm aneurysm (Fig. 1E, F). Complete coiling was performed for this lesion (Fig. 1G, H). In serial post-procedural computed tomography analyses, communicating hydrocephalus progressed and therefore this patient received a ventriculoperitoneal shunt. Finally, the patient was discharged in good clinical condition without neurological deficit, but slight organic brain syndrome.

\section{Clinical study 2}

This 46-year-old male patient presented initially with an incidental aneurysm at the middle cerebral artery bifurcation on the right side. Computed angiography and DSA revealed a two-lobulated 4 × 6 mm aneurysm (Fig. 2A, B) and endovascular treatment in balloon remodeling technique was recommended. An intraprocedural rupture occurred during endovascular procedure that could be successfully managed by further GDCs and angiographically complete occlusion was achieved (Fig. 2C, D). Direct post pro- 

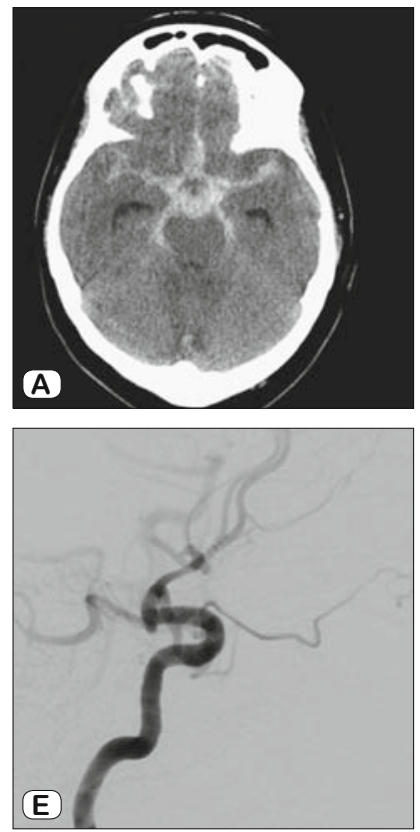
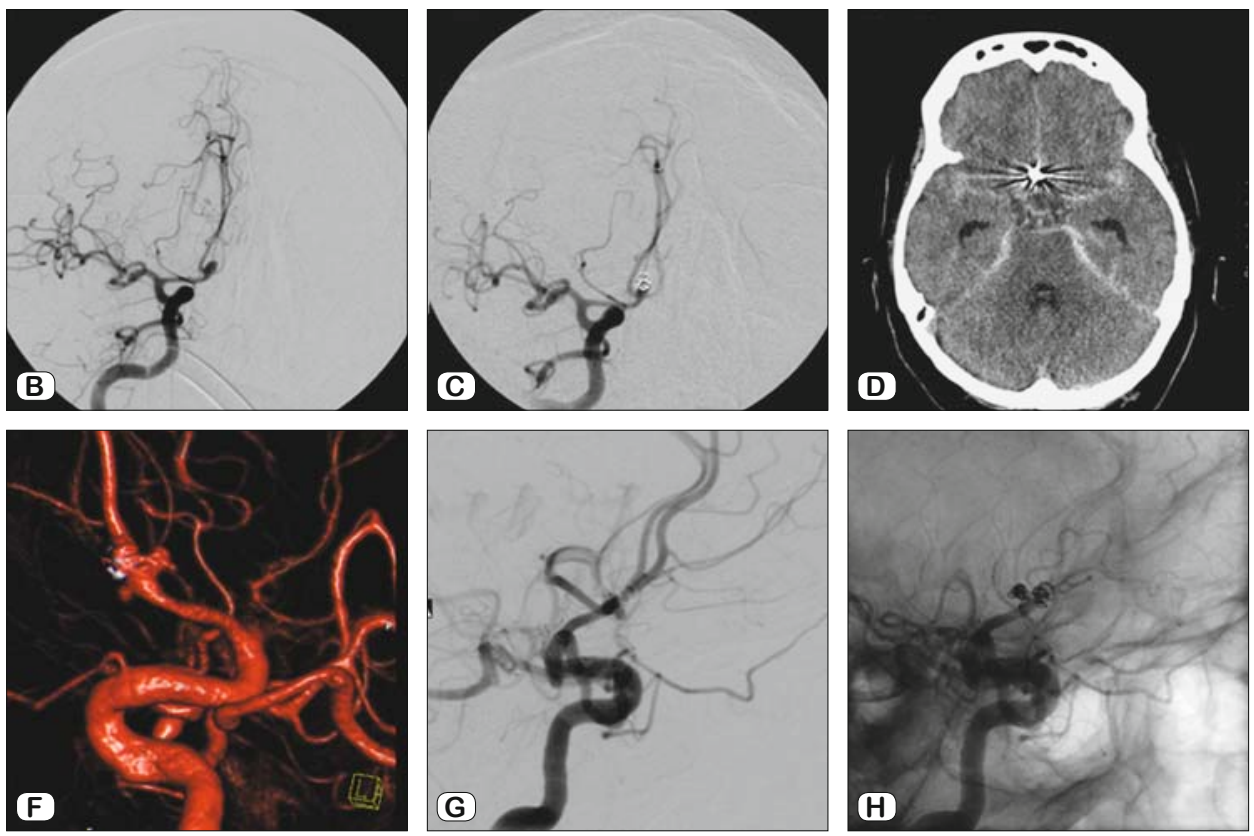

Fig. 1. A patient with subarachnoideal hemorrhage (A) and re-hemorrhage after ten years, due to rupture of an aneurysm of the anterior communication artery (Case 1). Digital subtraction angiography before (B) and after first endovascular coil occlusion (C). After ten years, this patient presented with rehemorrhage (D). Digital subtraction angiography (E) including 3D reconstruction (F) revealed a new 2x3mm aneurysm at the same location. Complete endovascular coil occlusion was achieved $(G, H)$.
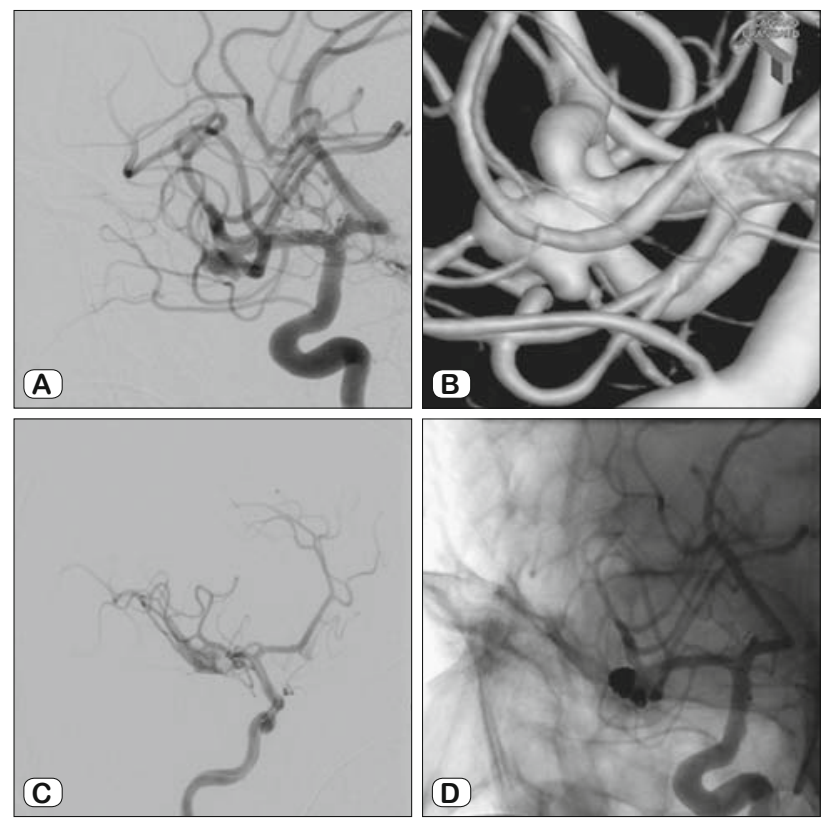

Fig. 2. A patient with an incidental aneurysm at the middle cerebral artery bifurcation (Case 2). Digital subtraction angiography (A) with 3D reconstruction (B). During endovascular coiling in balloon remodeling technique intraprocedural rupture occurred and could be successfully managed by further coiling. Angiographically, the aneurysm was completely occluded (C, D).

cedural CT-scan revealed hyperdense blood and contrast agent in the subarachnoid space and an intracerebral hematoma on the right side (Fig. 3A). Due to progressive brain swelling (Fig. 3B) with midline shift we decided to perform a right osteoclastic pterional craniotomy. Intraoperatively, the aneurysm appeared to be almost completely coiled. Only at the base, a small-uncoiled part was left (Fig. 3C, D). After temporary clipping of the M1 segment, all coils were removed and the aneurysm was completely clipped (Fig. 3E, F). Finally, the patient was discharged in good clinical condition with slight hemiparesis and organic brain syndrome.

\section{Clinical study 3}

This 63-year-old female patient presented with an incidental 6 mm aneurysm (Fig. 4A) at the middle cerebral artery bifurcation on the right side. Successful microsurgical clipping was performed. Another angiographic occult small lesion (Fig. 4B, C, D, E) was found at the base during surgery and was treated with another clip (Fig. 4F) to avoid its progression.

\section{Discussion}

Endovascular coiling has become a standard treatment and is more frequently considered to be a first-line treatment of intracranial aneurysms. The most frequent periprocedural complications are thromboembolic events and intraprocedural rupture (5). The rate of intraoperative ruptures ranges from $1 \%$ to $8.7 \%$ and can be managed either with further endovascular coiling or surgical clipping depending on situation (6).

In a recent meta-analysis, aneurysm recurrence after endovascular coiling was detected in $20.8 \%$, while in $10.3 \%$ retreatment 

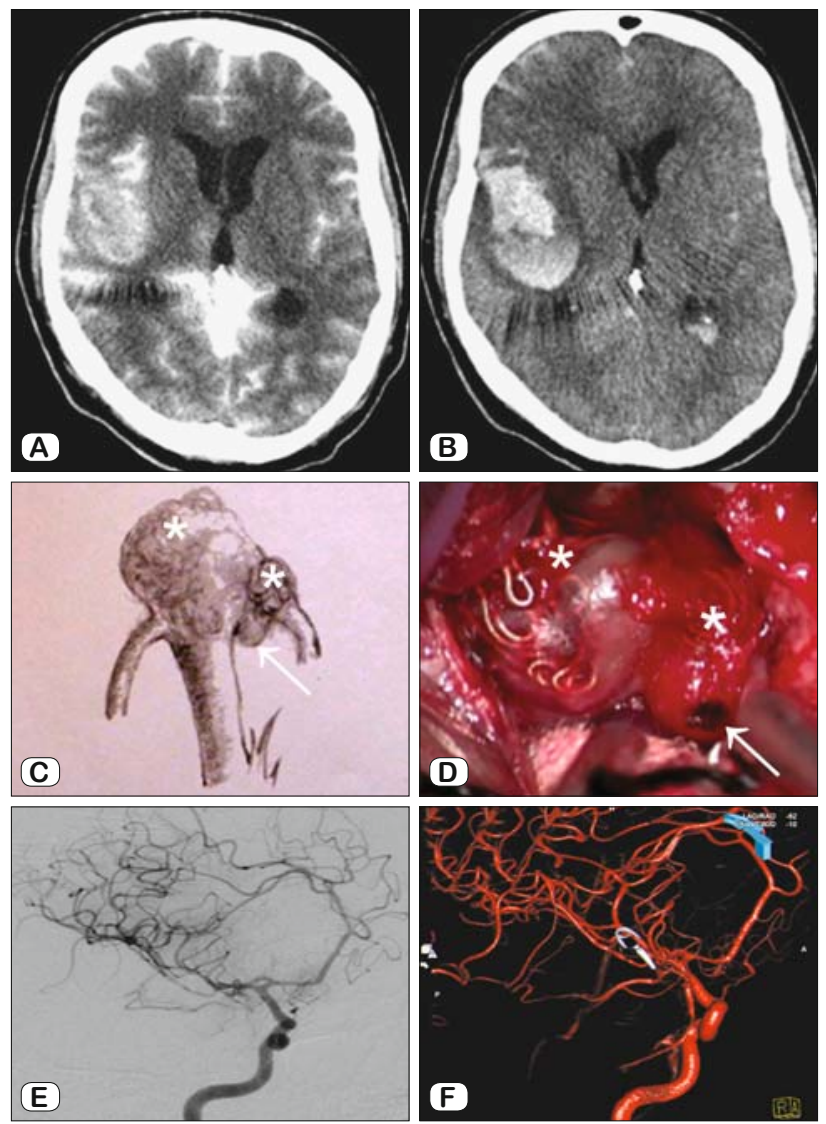

Fig. 3. A patient with an incidental aneurysm at the middle cerebral artery bifurcation (Case 2). Postprocedural computed tomography revealed a hematoma (A) and after two days a progressive brain swelling with midline shift (B). Therefore, a right pterional craniotomy and clipping of the aneurysm was performed. Anatomical sketch (C) and intraoperative view (D) during microneurosurgical operation. White stars: coiled part of the aneurysm; white arrows: uncoiled part of the aneurysm (C, D). Complete occlusion as demonstrated with postoperative digital subtraction angiography $(E)$ with $3 D$ reconstruction $(F)$ was performed.

was required (7). However, from the clinical point of view, it is essential that patients that are at risk of hemorrhage have to be provided with individualized treatment strategy.

The Cerebral Aneurysm Rerupture after Treatment Study (CARAT) has demonstrated, that both endovascular coiling and clipping provide effective protection against rebleeding within the first year, with lower rehemorrhage or retreatment rates after clipping. Rerupture rates after one year were extremely low with only one event in the coiling group (8). However, better short term clinical results are achieved with endovascular coiling according to the ISAT and BRAT study $(1,9,10)$, although this effect is lowered after 3 years of follow-up (11). The CARAT study further demonstrated that the initial occlusion degree is strongly associated with the risk of rerupture (12). Especially, when $>90$ $\%$ occluded, coiled aneurysms after 6 months of follow-up have a low cumulative incidence $(0.4 \%)$ for recurrent subarachnoid hemorrhage within the first 8 years (13). In another series, the incidence of aneurysms that repeatedly reopen after adequate coiling, and require multiple interventions is $1.5 \%$ (14). Bryne et al demonstrated, that stable remnants have a significantly lower rebleeding rate as compared to unstable aneurysms (0.4\% vs $7.9 \%$ ) (15).

Here, we present a rare case of rehemorrhage after 11 years (case 1) in a nearly occluded aneurysm with a stable base remnant. The fact that the aneurysm re-grew after 10 years might have two implications.

Firstly, the pathology at the base might have been underestimated under follow-up MR angiography studies. Although those aneurysms were angiographically considered as $100 \%$ occluded, upon histopathological analysis, tiny open spaces between the coils and incomplete membrane organization at the neck were detected, (16). In our case 2, where an intraprocedural rupture could be initially managed by further coiling, the intraoperative view during clipping revealed the real situation with an uncoiled part of the aneurysm at the base. Similarly, in our case 3, only direct intraoperative view revealed an additional small lesion at the base. Especially multiple lesions or a daughter sac (17) might have increased the risk of rupture even in incidental cerebral aneurysms. Note that the aneurysm in case 2 has initially been angiographically considered to be completely occluded. Underestimation of the initial occlusion status might therefore be in part responsible for both, early and late aneurysm recurrences or ruptures, regardless of the initial treatment strategy. Another study highlights the importance of independent evaluation of angiographic results after endovascular coiling. Independent reviewers judged the initial and 1-year angiographic occlusion states as being significantly worsened as compared to local physicians (4). Furthermore, it is not
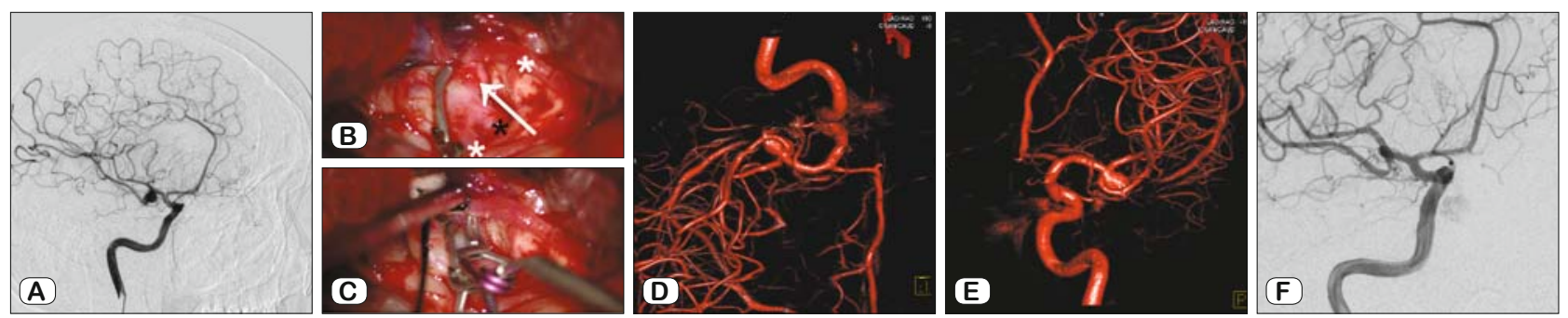

Fig. 4. A patient with an incidental aneurysm at the medial bifurcation (Case 3). Preoperative digital subtraction angiography (A). Intraoperatively, after clipping of the aneurysm an additional small lesion (white arrow) was detected at the base. White stars: M2, black star: M1 (B). This lesion was treated with another clip to avoid its progression (C). Preoperative 3D reconstruction (D, E). Postoperative digital subtraction angiography verified the complete occlusion of aneurysm (F). 
absolutely clear as to for how long the aneurysm after endovascular procedures needs to be followed up (18). In the case of adequately occluded aneurysms upon control DSA after six months, further MR angiographies are considered to be an appropriate follow-up imaging tool (19). To date, our current policy is to perform a DSA after 6 months followed with serial MR angiographies, even in case of stable results up to 10 years.

Secondly, when considering the base to be the locus minoris resistentiae (20), a stable base remnant or even a totally occluded aneurysm still might be vulnerable to aneurysm regrowth in the long term. Especially multiple lesions might indicate that the base should be considered a weak point. The incidence of late reopenings of aneurysms that are adequately coiled after six months is extremely low (18). In general, the mechanisms of recurrence are incompletely understood. Hasan et al. provide evidence that aneurysm sac growth might be the etiology after successful coiling (21). Other mechanisms involved are coil compaction, fundal migration (20) or coil extrusion (22). However, in these cases the exact mechanism can be only assessed during surgery (20). As demonstrated in our cases 2 and 3 , small lesions at the base that are undetected by DSA could be the source of future aneurysms.

In various studies, it could be demonstrated, that aneurysm rupture and growth are mediated by angiogenesis, inflammatory cell infiltration $(23,24)$ or extracellular matrix degeneration (25) in the aneurysmal wall. Further, immunohistochemical analyses revealed differences in structural protein and vascular growth factor expressions in normal artery, as well as unruptured and ruptured aneurysm walls (26). In line with these findings, another study demonstrated that the recurrence might be affected by the rupture status with more events in previously ruptured aneurysms (27).

These findings suggest, that on the one hand the mechanical properties of the coil complex and on the other hand the mechanisms on an inflammatory/biological basis in the aneurysms wall and base might contribute to aneurysm recurrence and hemorrhage.

In conclusion, in our clinical studies, the pathology at the base has been underestimated upon DSA or MR angiography. The incidence of angiographic occult residuals is unknown. However, very small lesions might only be visible during microneurosurgery. As demonstrated, very late rebleedings occur even in long-term stable base remnants that are widely regarded as benign lesions. As the follow-up period of endovascular-coiled aneurysms is increasing, it is possible that stable remnants can become a clinically relevant phenomenon.

\section{References}

1. Molyneux A, Kerr R, Stratton I, Sandercock P, Clarke M, Shrimpton J, Holman R, International Subarachnoid Aneurysm Trial Collaborative G: International Subarachnoid Aneurysm Trial (ISAT) of neurosurgical clipping versus endovascular coiling in 2143 patients with ruptured intracranial aneurysms: a randomised trial. Lancet 2002; 360 (9342): 1267-1274.
2. Crobeddu E, Lanzino G, Kallmes DF, Cloft HJ: Review of 2 decades of aneurysm-recurrence literature, part 1: reducing recurrence after endovascular coiling. Amer J Neuroradiol 2013; 34 (2): 266-270.

3. Notarianni C, Vannemreddy P, Caldito G, Bollam P et al. Congenital hydrocephalus and ventriculoperitoneal shunts: influence of etiology and programmable shunts on revisions. J Neurosurg Pediat 2009; 4 (6): 547-552.

4. Taki W, Sakai N, Suzuki H. Prospective Registry of Subarachnoid Aneurysms Treatment: Importance of independent evaluation of initial anatomic results after endovascular coiling for ruptured cerebral aneurysms. J Clin Neurosci 2013; 20 (4): 527-531.

5. Pierot L, Barbe C, Spelle L, investigators A. Endovascular treatment of very small unruptured aneurysms: rate of procedural complications, clinical outcome, and anatomical results. Stroke 2010; 41 (12): 2855-2859.

6. Zhang Y, Li G, Cai Y et al. Rupture during the endovascular treatment of intracranial aneurysms: outcomes and technical aspects. Acta Neurochir 2013; 155 (4): 569-577.

7. Ferns SP, Sprengers ME, van Rooij WJ et al. Coiling of intracranial aneurysms: a systematic review on initial occlusion and reopening and retreatment rates. Stroke 2009; 40 (8): e523-529.

8. Investigators C. Rates of delayed rebleeding from intracranial aneurysms are low after surgical and endovascular treatment. Stroke 2006; 37 (6): 1437-1442.

9. McDougall CG, Spetzler RF, Zabramski JM et al. The Barrow Ruptured Aneurysm Trial. J Neurosurg 2012, 116 (1): 135-144.

10. Molyneux AJ, Kerr RS, Yu LM et al. International Subarachnoid Aneurysm Trial Collaborative G: International subarachnoid aneurysm trial (ISAT) of neurosurgical clipping versus endovascular coiling in 2143 patients with ruptured intracranial aneurysms: a randomised comparison of effects on survival, dependency, seizures, rebleeding, subgroups, and aneurysm occlusion. Lancet 2005; 366 (9488): 809-817.

11. Spetzler RF, McDougall CG, Albuquerque FC et al. The Barrow Ruptured Aneurysm Trial: 3-year results. J Neurosurg 2013; 119 (1): 146-157.

12. Johnston SC, Dowd CF, Higashida RT, Lawton MT, Duckwiler GR, Gress DR, Investigators C. Predictors of rehemorrhage after treatment of ruptured intracranial aneurysms: the Cerebral Aneurysm Rerupture After Treatment (CARAT) study. Stroke 2008; 39 (1): 120-125.

13. Schaafsma JD, Sprengers ME, van Rooij WJ et al. Long-term recurrent subarachnoid hemorrhage after adequate coiling versus clipping of ruptured intracranial aneurysms. Stroke 2009; 40 (5): 1758-1763.

14. van Rooij WJ, Sprengers ME, Sluzewski M, Beute GN. Intracranial aneurysms that repeatedly reopen over time after coiling: imaging characteristics and treatment outcome. Neuroradiology 2007; 49 (4): 343-349.

15. Byrne JV, Sohn MJ, Molyneux AJ, Chir B. Five-year experience in using coil embolization for ruptured intracranial aneurysms: outcomes and incidence of late rebleeding. J Neurosurg 1999; 90 (4): 656-663.

16. Bavinzski G, Talazoglu V, Killer $M$ et al. Gross and microscopic histopathological findings in aneurysms of the human brain treated with Guglielmi detachable coils. J Neurosurg 1999; 91 (2): 284-293.

17. Morita A, Kirino T, Hashi $K$ et al. The natural course of unruptured cerebral aneurysms in a Japanese cohort. New Engl J Med 2012; 366 (26): 2474-2482. 
18. Ferns SP, Sprengers ME, van Rooij WJ et al. Late reopening of adequately coiled intracranial aneurysms: frequency and risk factors in 400 patients with 440 aneurysms. Stroke 2011; 42 (5): 1331-1337.

19. Crobeddu E, Lanzino G, Kallmes DF, Cloft HJ. Review of 2 decades of aneurysm-recurrence literature, part 2: Managing recurrence after endovascular coiling. Amer J Neuroradiol 2013; 34 (3): 481-485.

20. Dorfer C, Gruber A, Standhardt H, Bavinzski G, Knosp E. Management of residual and recurrent aneurysms after initial endovascular treatment. Neurosurg 2012; 70 (3): 537-553.

21. Hasan DM, Nadareyshvili AI, Hoppe AL, Mahaney KB, Kung DK, Raghavan ML. Cerebral aneurysm sac growth as the etiology of recurrence after successful coil embolization. Stroke 2012; 43 (3): 866-868.

22. Pogady P, Fellner F, Trenkler J, Wurm G. Surgical management of an ACM aneurysm eight years after coiling. Minim Invas Neurosurg 2007; 50 (2): 102-105.
23. Hoh BL, Hosaka K, Downes DP et al. Stromal cell-derived factor-1 promoted angiogenesis and inflammatory cell infiltration in aneurysm walls. J Neurosurg 2014; 120 (1): 73-86.

24. Tulamo R, Frosen J, Hernesniemi J, Niemela M. Inflammatory changes in the aneurysm wall: a review. J Neurointerven Surg 2010; 2 (2): 120-130.

25. Penn DL, Witte SR, Komotar RJ, Sander Connolly E, Jr. The role of vascular remodeling and inflammation in the pathogenesis of intracranial aneurysms. J Clin Neurosci 2014; 21 (1): 28-32.

26. Kilic T, Sohrabifar M, Kurtkaya $O$ et al. Expression of structural proteins and angiogenic factors in normal arterial and unruptured and ruptured aneurysm walls. Neurosurg 2005; 57 (5): 997-1007.

27. Cognard C, Weill A, Spelle L, Piotin M, Castaings L, Rey A, Moret J. Long-term angiographic follow-up of 169 intracranial berry aneurysms occluded with detachable coils. Radiology 1999; 212 (2): 348-356.

Received June 14, 2015. Accepted July 3, 2015. 